\title{
The neuroendocrinological
}

sequelae of stress during brain

development: the impact of child

abuse and neglect

\author{
A Panzer \\ Department of Physiology, School of Health Sciences, University of Pretoria, Pretoria, South Africa
}

\begin{abstract}
Severe stress during the sensitive periods of neurodevelopment, (which include the prenatal period, infancy, childhood and adolescence), has a long-lasting organizing effect on the brain and stress axes. Child abuse and neglect thus exert a cumulative harmful effect on neuroendocrinological development, which persists into adulthood. It is not merely the memory of the trauma which leaves a mark, but rather the effect on neurodevelopment which negatively influences the ability of adult survivors of childhood maltreatment to cope with current stressors. The victims of child abuse and neglect are likely to maltreat their own children and so perpetuate the intergenerational transmission of child maltreatment. In this paper relevant normal brain development is first summarized. Child abuse/neglect is next discussed with detailed reference to the aberrant neuroendocrinological development that is known to occur. We specifically examine effects on the hypothalamo-pituitary-adrenal and central noradrenergic-sympathoadrenomedullary stress axes and other neurotransmitter systems before turning to changes described in the cerebral volumes, corpus callosum and cortical hemispheres, prefrontal cortex and amygdalae, superior temporal gyrus, hippocampus as well as the cerebellar vermis.
\end{abstract}

Keywords: Child abuse; Child neglect; Neuroendocrinology; Brain

Submitted: $27-11-2006$

Accepted: 18-05-2007

\section{Introduction}

Child abuse and/or neglect have devastating consequences, not only for the present, but also for the future of the child. This is due to the fact that babies are born with exceedingly immature brains and undergo major processes of neuroendocrinological maturation. During this developmental period, the brain and stress axes are exceptionally vulnerable to environmental influences. Severe stress during the early years leads to longterm changes, affecting the child not only when he or she is grown up, but also his/her children, who are much more likely to be abused and/or neglected. The intergenerational transmission

\section{Correspondence:}

Dr A Panzer

Department of Physiology, School of Health Sciences, University of Pretoria. PO Box 2034, Pretoria 0001, South Africa

email: annie.panzer@gmail.com of child maltreatment is a vicious cycle. Any successful intervention will most likely not only benefit the targeted individual, but also his or her future offspring.

In this paper relevant normal brain development is first summarized. The relevance of the "nature versus nurture" debate is challenged with examples of how the environment shapes the genetic make-up. Thereafter, child abuse/neglect is discussed with detailed reference to the aberrant neuroendocrinological development that is known to occur. The paper concludes with some comments on intervention.

Important aspects of neuroendocrinological development At birth, babies are the most helpless of all mammals. Human infants have a protracted period of development compared to other species. ${ }^{1}$ This lengthened time for learning allows for the development of a far superior intellect (compared to other animals). However, the plasticity and adaptability of the young human brain also makes it very vulnerable to adverse 
environmental influences. Before discussing the effects of child maltreatment, it is pertinent to briefly discuss what is known about human brain development in more favorable circumstances

\section{Growth spurts, gray/white matter ratio and sensitive periods}

The greatest growth spurt of the brain begins in utero. ${ }^{1}$ Babies are born with large brains, which account for more than half of their metabolic needs. ${ }^{2}$ The genetically driven overproduction of synapses reaches it peak at 24 months, at which time these barely two-year olds have double the amount of synapses compared to adults! ${ }^{1}$ On the cellular level, the proportion of cerebral gray matter to white matter decreases progressively after the first few years. ${ }^{3}$ Gray matter decreases due to reductions in synaptic density as a result of pruning, while white matter increases as a result of increased myelination of newly formed neuronal networks. ${ }^{3}$ Both pruning and myelination are very susceptible to environmental influences. ${ }^{1,3}$

Sensitive periods have been identified during which the developing brain is especially responsive to environmental input. ${ }^{1}$ Neurotrophins are one putative mechanism by which the environment can shape the brain. Neurotrophins regulate neuronal survival and differentiation. The secretion of neurotrophins is regulated by neuronal activity, which is directly related to environmental input. ${ }^{1}$ Two facets of these environmentally dependent brain maturational processes have been described, i.e. experience-expectant, and experiencedependent processes. ${ }^{1}$ Experience-expectant deficits occur when failure of environmental stimulation during critical periods of brain development may lead to permanent problems. ${ }^{1} \mathrm{~A}$ relatively recent example would be infants in Romanian orphanages, where a single caretaker was responsible for up to 60 babies. ${ }^{3}$ The Romanian children who were adopted were found to have delayed growth, gross motor skills and language, as well as suffer from inattention and hyperactivity, attachment disorder behavior, autistic-like behaviors and deficits in social functioning. ${ }^{3}$ These poor outcomes were associated with the period of institutionalization. ${ }^{3}$ The other type of environmentally dependent brain maturational processes is known as experiencedependent. In these experience-dependent processes, new synapses are formed in response to environmental input. ${ }^{1}$ This accounts for the learning of various skills. In general, neglect leads to deprivation of input needed by the infant brain at times of experience-expectant maturation, while abusive experiences affect brain development at experience-dependent stages. ${ }^{1}$ Unfortunately, young children often experience both abuse and neglect.

\section{Prefrontal cortex and corpus callosum}

The sequence of neurodevelopment is remarkably stable, bearing proof of its genetic predetermination. Two main drives in brain development are firstly the maturation of the prefrontal cortex, which controls the limbic centers and enables executive cognitive functions and secondly the integration of the hemispheres through further development of the corpus callosum. Both of these processes start in utero and are only fully completed in the third decade of life. ${ }^{3}$ The prefrontal cortex permits the control of emotions and behavior and is thus responsible for the ability to think things through before reacting. ${ }^{3}$ The corpus callosum enables hemispheric integration and exhibits the most dramatic increase in myelination during neurodevelopment. ${ }^{3}$ Environmental influences strongly affect both the maturation of the prefrontal cortex and the development of the corpus callosum, as will be discussed in more detail later.

\section{Stress axes}

Stress is defined as "a stimulus or experience that produces a negative emotional reaction or affect, including fear and a sense of loss of control". I Child abuse and neglect are potent sources of stress. ${ }^{1}$ The stress response is a physiological coping response, which involves the hypothalamic-pituitary-adrenal (HPA) axis, the central noradrenergic sympathomedullary system (CNA-SAM) and other neurotransmitters. ${ }^{1}$ The purpose of the stress response is to mobilize resources with the aim of increasing the individual's chance of survival. Inappropriate responsiveness of the stress system may contribute to various endocrine, metabolic, autoimmune and psychiatric disorders. ${ }^{4}$ The development and severity of these disorders depend on genetic vulnerability, environmental factors and the timing of the stressful event(s), (because prenatal life, infancy, childhood and adolescence are critical periods characterized by increased vulnerability to stress). ${ }^{4}$ The two central stress axes, i.e. the hypothalamo-pituitary adrenal axis (HPA) and central noradrenergic sympathoadrenomedullary axis (CNA-SAM), are briefly reviewed before considering a few other neurotransmitter systems.

\section{- HPA-axis}

When a stressor is perceived through the senses, the amygdalae activate the paraventricular nucleus of the hypothalamus (PVNh), which releases corticotropin releasing hormone $(\mathrm{CRH})$. $\mathrm{CRH}$ then acts on the anterior pituitary, stimulating the release of adrenocorticotrophic hormone (ACTH), which in turn causes the adrenal glands to release cortisol. Increased levels of cortisol then further stimulate the amygdala to act on the PVNh to further increase cortisol release. This relationship between the amygdala on the one hand and the HPA-axis on the other thus represents a positive feedback system, which, if allowed to continue, would have dire physiological, as well as psychological, consequences. In the short term, the benefits include: a) mobilization of energy, b) release of catecholamines with resultant increase in cardiovascular activity, c) increase in alertness, d) promotion of stress-induced analgesia, and e) suppression of nonessential immediate functions like growth, reproduction, immune functions and digestion. ${ }^{4}$ Persistent activation of the HPA system is associated with immune deficiency, cognitive impairment, inhibited growth, delayed sexual maturity, psychological maladjustment, essential hypertension and visceral obesity. 2,4,5

The HPA-axis is controlled by cortisol providing negative feedback to the pituitary, the hypothalamus and the hippocampus with the hippocampus representing the primary negative feedback regulatory mechanism. ${ }^{6}$ The density of glucocorticoid receptors necessary for negative feedback and thus termination of the stress response is dependent on environmental influences. Tactile stimulation derived from the mother increases glucocorticoid receptor expression in the hippocampus, thus decreasing HPA activity. ${ }^{4}$ Conversely, maternal deprivation in infancy is associated with enhanced neural CRF gene expression and increased stress 
reactivity in adulthood. ${ }^{4}$ With severe or relentless stress, the stress hormones lead to glucose depletion in the hippocampal cells, making them sensitive to damage by excess glutamate. ${ }^{7}$ The control of the HPA-axis thus becomes compromised.

Early life experiences enduringly influence the development of central CRF systems, which in turn mediate the expression of behavioral/emotional, autonomic and endocrine responses to stress. ${ }^{4}$ Animal experiments suggest that maternal separation-induced changes in CRF systems might control both noradrenergic and serotonergic responses to stress. ${ }^{4}$

\section{- CNA-SAM axis}

$\mathrm{CRH}$ released by the PVNh also stimulates the locus coeruleus to release noradrenaline, which instantly gives rise to the bodily response of the fight-or-flight or freeze response. ${ }^{1}$

\section{- Other neurotransmitter systems}

Both the $\mathrm{CRH}$ and the noradrenergic system receive stimulatory innervation from the serotoninergic and cholinergic systems and inhibitory input from GABA and the opioid peptide neuronal systems as well as from the end product of the HPA axis, glucocorticoids. ${ }^{4}$ The mesocortical dopaminergic system involves dopaminergic neurons of the ventral tegmentum that send projections to the prefrontal cortex. ${ }^{4}$ Activation of the prefrontal cortex is associated with inhibition of the stress system. ${ }^{4}$

In summary, following emotional stressors the amygdala can stimulate the HPA-axis, CNA-SAM axis and the mesocorticolimbic dopaminergic system. ${ }^{4}$ Inhibition of the stress response is achieved by cortisol binding to $\mathrm{CRH}$ receptor sites in the hippocampus, the GABA and opioid peptide systems as well as activation of the prefrontal cortex by the mesocortical dopaminergic system. ${ }^{4}$

Neuroendocrinological development occurs with a genetic drive in an influencing environment. It is apparent that the "nature" versus "nurture" debate was simplistic, as in many cases both the genetic predisposition and the environment are inextricably intertwined. A discussion of environmental influences and then some genetic predispositions known to play a role in development follows.

\section{Environmental influences}

Environmental influences are varied and far-reaching. Prenatal factors have been shown to affect the developing brain for years to come, as demonstrated by the following two examples, the one describing maternal stress and the other maternal substance abuse. Stress during pregnancy was found to predict delayed motor and mental development in the infant. ${ }^{8}$ A recent study, which tracked the development of children who were exposed in utero to a natural disaster, found impairments in the cognitive and language development at age $2 .{ }^{9}$ The most severe impairments were found if the child was exposed during midgestation. ${ }^{9}$ Maternal substance disorders during pregnancy are associated with attentional problems in the child, probably by causing adverse effects on the developing stress systems and brain maturation of the fetus. ${ }^{3}$

Apart from the prenatal factors mentioned above, environmental influences on postnatal neurodevelopment include poverty, nutrition, stress, availability of health care and educational opportunities. Abused and neglected children often live in a poor socio-economic environment, which is associated with less optimal nutrition, poorer health care, fewer educational opportunities, domestic violence and parental substance disorders. ${ }^{1,3}$ The degree of support the child receives can either buffer or exacerbate the environmental stressors he or she is exposed to. ${ }^{1}$ For example, in domestic violence shelters, child post-traumatic stress disorder (PTSD) symptoms were associated with the amount of physical violence the child was exposed to, whether experienced or witnessed. ${ }^{10}$ In contrast, child behavioral problems were related to mother anxiety and anger. ${ }^{10}$ Abused mothers demonstrated less maternal warmth, which was associated with observed child antisocial behavior. ${ }^{10}$

\section{Genetic influences}

It is generally thought that whereas genes are responsible for potential (setting the limits), the environment determines how much of the potential will be reached. ${ }^{11}$ Child maltreatment thus prevents children from realizing their genetic cognitive potential, and may trigger the development of diseases they are genetically predisposed to. ${ }^{12}$ Gender is also important, as boys are less resilient to maltreatment than girls. ${ }^{3}$ Even if the age of onset, duration of abuse, similar type of abuse and the length of time since disclosure of abuse are controlled for, boys show more evidence of adverse brain development, e.g. delayed corpus callosum myelination (see later). ${ }^{3}$ Three examples where the environment was shown to moderate the genetic make-up include the correlation between IQ and academic achievement, the relation between a polymorphism in the monoamine oxidase A (MAOA) enzyme and antisocial behavior and the biological risk of schizophrenia.

IQ is strongly related to the amount of frontal gray matter, as measured by structural MRI, with both being highly heritable. ${ }^{11}$ Among children from high socio-economic status, IQ is related to academic achievement. ${ }^{12}$ However, among children from a poor socio-economic environment, their IQ is almost irrelevant in predicting their academic success. ${ }^{12}$ The environment in these children, probably because they tend to have fewer educational opportunities and suffer more from psychological stress, is "the single greatest contributor to impaired academic learning". ${ }^{7}$ The environment thus moderates the genetic effects. ${ }^{13}$

Another recently discovered genotype-environment interaction is a polymorphism in the monoamine oxidase $A$ (MAOA) enzyme, which predisposes individuals to antisocial behavior only in the presence of an unfavorable rearing environment. ${ }^{3}$ Maltreated children with a genotype conferring high levels of MAOA were less likely to develop antisocial problems. ${ }^{3}$ Maltreated males with low MAOA levels were three times more likely to be convicted of a violent crime by age 26 than maltreated males with the protective genotype. ${ }^{14}$

In an adoption study it was found that the biological offspring of a parent with schizophrenia were at risk of developing schizophrenia only when adopted into a dysfunctional family. ${ }^{15}$

Having reviewed the brain, the stress response and some environmental and genetic influences, the issue of to child maltreatment is discussed giving first give an introduction on child abuse and neglect and then consider its effects on the brain and stress systems. 


\section{Child abuse and neglect: a general introduction}

According to Glaser, "child abuse and neglect are (wo)manmade phenomena which adversely affect a child's development and sometimes survival, and which should, at least in theory, be preventable". ${ }^{1}$ Child abuse (or neglect) is defined as an act (or omission of care), which creates an actual or potential physical or mental risk to a child under 18 years of age., ${ }^{1,3}$ Child abuse is subdivided in to physical, emotional or sexual abuse.1,3 These abusive acts can occur once, repeatedly or can be a pattern of interaction in a dysfunctional relationship with a caregiver. ${ }^{1,3}$ Child neglect is subdivided into physical, emotional, medical or educational neglect. ${ }^{3}$ Unfortunately various subtypes of abuse and neglect often coexist. ${ }^{1,3}$ Outcome is dependent on the subtype(s), severity, frequency and chronicity of abuse and neglect, as well as child characteristics like age, gender, temperament and any disabilities. ${ }^{1,3}$ The age of onset of child maltreatment was found to predict long-term mental health outcome, as would be expected with stressors impacting negatively on neurodevelopment. ${ }^{16}$ Furthermore, the maltreated child and their caregiver often live in poverty and under difficult social circumstances. ${ }^{1}$ Children are likely to suffer other adversities such as prenatal exposure to substances, witnessing domestic violence, poor nutrition and lack of educational opportunities. ${ }^{3}$ The abusing or neglecting caregiver is likely to have suffered maltreatment in his or her own childhood. ${ }^{1}$ This leads to a vicious cycle of maltreatment, which can, however, in principle be stopped if the individual can talk about the adverse experiences and integrates them in a coherent fashion, (which is the purpose of intervention and psychotherapy). ${ }^{1}$

General effects of child abuse and neglect include social, emotional, behavioral, cognitive and emotional problems as well as frank psychopathology, both in later childhood and adulthood. ${ }^{1}$ However, it is not possible to predict accurately the outcome of certain adverse experiences as other factors, e.g. ego resiliency and support from other persons may limit the damage. ${ }^{1}$

The reason why child maltreatment has such devastating consequences is that the child is developing, the brain is growing, and the set points for activation of the stress axes are programmed. 1,3 Severe stress during this period influences the formation of the brain and has long-term consequences. One mechanism by which abuse and neglect shape the brain is through high levels of stress hormones. Abuse and neglect cause very high levels of stress in children as their whole existence is in immediate or potential danger., 1,3

\section{Effects of child abuse and neglect on the brain and stress axes}

Brain regions that are particularly susceptible to the effects of early stress have one or more of the following in common: a) extended postnatal development, b) a high concentration of glucocorticoid receptors and c) some extent of postnatal neurogenesis. ${ }^{17}$ Chronic abuse and neglect may have a cumulative, damaging effect on neurodevelopment. ${ }^{2}$ These effects occur on multiple levels, from neurohumoral to structural.

\section{The major neurohumoral changes associated with child abuse and neglect:}

- CNA-SAM axis: noradrenaline Intense anxiety, whether caused by a real or perceived threat, activates the locus coeruleus and the sympathetic nervous system, releasing noradrenaline. ${ }^{3,7}$ Noradrenaline triggers the fight-or-flight or freeze reaction with a resultant increase in heart rate, blood pressure, metabolic rate and alertness. ${ }^{3}$ Direct and indirect effects of this activation include increases in catecholamine turnover in the brain, the sympathetic nervous system and the adrenal medulla. ${ }^{3}$ Childhood maltreatment is associated with a life-long increase in the sensitivity of the noradrenergic system, possibly due to a decrease in the (inhibitory) alpha-2 autoreceptor of the locus coeruleus, which results in increased noradrenergic reactivity.18

\section{- Dopamine}

The amygdala stimulates dopaminergic inputs to the medial prefrontal cortex, with the result of heightened attention to cope with the stressor. ${ }^{3}$ However, chronic stress may produce excessive prefrontal dopamine, which may then impair prefrontal cortical function. ${ }^{3}$ This may result in inattention, hypervigilance, psychotic symptoms and problems with memory and learning, ${ }^{3,11}$ Abnormal regulation of the mesocorticolimbic dopaminergic system has also been implicated in dysthymia, novelty-seeking, and addictive behaviors. ${ }^{4}$

\section{- Serotonin}

The serotonin system is regarded as a master control neurotransmitter of complex neuronal communication. ${ }^{3}$ It is a stress response system that activates both anxiogenic and anxiolytic pathways. ${ }^{3}$ Serotonin is important for the control of emotions and behavior. ${ }^{3}$ Serotonin depletion may result in behaviors of learned helplessness, impulsivity and aggression. ${ }^{3}$

\section{- HPA axis}

In contrast to the autonomic nervous system, which can be activated by physical, mental and psychosocial tasks, the HPA axis seems more specific to a challenge, which incorporates social competence. ${ }^{19}$ Social competence is difficult, for other people are also constantly changing and have similar cognitive abilities. ${ }^{2}$ Traumatized children sustain cortisol elevations in response to social challenges longer than nonmaltreated children. ${ }^{2}$ Children who were maltreated have significantly higher cortisol levels compared to controls. ${ }^{2,3}$ Interestingly, the opposite holds true for previously maltreated adolescents and adults, who have lower cortisol levels than controls. ${ }^{1,3}$ With chronic stress, the HPA-axis is down regulated and cortisol levels return to normal. ${ }^{1,17}$ It is thought that down regulation occurs in response to a persistent chaotic environment; to prevent chronic arousal with associated excessive energy expenditure. ${ }^{5}$ This HPA-axis down regulation occurs in children who have suffered long-term abuse. ${ }^{1}$ It is possible that the increased cortisol levels at the time of abuse are neurotoxic to the hippocampus. ${ }^{2}$ This has been proven in rodents, where diminished maternal care is associated with a high cortisol level, which in turn causes DNA methylation of the glucocorticoid receptor in the hippocampus. ${ }^{2}$ Stress response hypoarousal has been described in individuals at risk for or with antisocial behavior disorder. ${ }^{5,14}$ Low basal cortisol has been found in antisocial children, adolescents and adults. ${ }^{5}$ Conversely, low arousal as identified with heart rate and variability, is a predictor of future antisocial behavior. ${ }^{5}$ Danger-seeking behavior may be 
an attempt to increase arousal, as low arousal is said to be 'uncomfortable'. 5

\section{The major structural and functional changes associated with child abuse and neglect:}

Structural changes occur through abnormal pruning, inhibited neurogenesis and delays in myelination. ${ }^{3}$ Oligodendrocytes make the myelin sheaths of neurons, i.e. white matter. ${ }^{16}$ High levels of stress hormones suppress the final mitosis of oligodendrocytes from precursors. ${ }^{20}$ Thus, the formation of white matter is impaired.

\section{- Cerebral volumes}

Cerebral volumes are smaller while the ventricles are larger. ${ }^{1}$ Earlier onset of abuse and longer duration of abuse correlated with smaller intracranial volume. ${ }^{3}$ There is a positive correlation between IQ measures and brain size.1,3

\section{- Cerebral cortex and corpus callosum}

The left hemisphere is specialized for language and analytical thought. ${ }^{16}$ The right hemisphere is dedicated to the perception and expression of emotion, especially negative emotion. ${ }^{16}$ Psychiatric inpatients have significantly more developed right than left hemispheres. ${ }^{16}$ The innervation patterns of neurotransmitter systems in the brain are lateralized and this is exacerbated by early stress. ${ }^{16}$ Right to left asymmetries in serotonin and dopamine projections to the amygdala and prefrontal cortex are correlated with anxiety. ${ }^{16}$ Child maltreatment is associated with delays in corpus callosum myelination. ${ }^{3.16}$ Reduced size of the corpus callosum has been associated with decreased communication between the cortical hemispheres, thus permitting the hemispheres to develop somewhat independently from one another. ${ }^{3,16}$ A recent study describes adult subjects who had suffered from child maltreatment, using the left hemisphere for a neutral school memory and the right hemisphere when thinking of a disturbing childhood memory. ${ }^{16}$ In contrast, adults who had not been abused employed both hemispheres for both tasks. ${ }^{16}$ Females generally have a greater bilateral linguistic capacity and reduced hemispheric dominance compared to males. ${ }^{16}$ Women who suffered abuse may switch between left and right polarized states, thus increasing affective instability and the risk for borderline personality disorder. ${ }^{16}$ In contrast, the strong left hemispheric specialization and diminished corpus callosum development in abused males may lead to the separation of analytical thought from affect and social awareness (in the right hemisphere) and may facilitate the development of alexithymia or antisocial personality disorder. ${ }^{16}$ The widely observed association between childhood maltreatment and self-injurious behavior may be mediated by alexithymia. ${ }^{21}$ Self-injurious behavior may be a way of expressing emotional pain and distress. ${ }^{21}$

\section{- Hippocampus}

Most studies find a reduction of hippocampal volumes. ${ }^{1,3,12,17}$ Mechanisms may include glucocorticoids potentiating the toxicity of excitatory amino acids, decreased brain derived neurotrophic factor, inhibition of neurogenesis or a combination of these factors. ${ }^{18}$ Symptoms may include the amnestic, dissociative, anxiogenic and disinhibitory aspects of PTSD. ${ }^{17}$ Therapeutic interventions may enhance hippocampal neurogenesis. ${ }^{3,12}$

- Amygdala, temperolimbic seizures and the prefrontal cortex Amygdalae are sensitive to the development of kindling. ${ }^{17}$ Kindling is a process in which repetitive sporadic neuronal stimulation produces greater and greater changes in the excitability of those neurons, ultimately resulting in spontaneous electrical discharges, or seizures. ${ }^{17}$ Kindling in irritable foci in the amygdala may lead to sporadic behavioral loss of control and impulsive violence. ${ }^{17}$ Early stress is also related to a long-term modification in the subunit composition of the GABA-A supramolecular complex in the amygdala. ${ }^{17}$ This may cause 'limbic irritability', i.e. perceptual distortions, brief hallucinatory events, motor automatisms and dissociative phenomena. ${ }^{17}$ Children with a history of abuse have EEG abnormalities in the frontotemporal region, predominantly in the left hemisphere. 1,17,20 Infants of depressed mothers also have decreased left frontal EEG activity. ${ }^{1}$ EEG abnormalities may be a significant risk factor for suicidal ideation or attempts. ${ }^{17}$ Epileptic patients have a substantially higher suicide risk than patients with other medical disorders that produce a similar risk of disability. ${ }^{17}$ The EEG abnormalities seen in trauma survivors are associated with increased frequency of violence..$^{14}$

Severe stress manifesting as chronic amygdala activation may impair the development of the anterior cingulated cortex, a region of the medial prefrontal cortex, which is involved in the extinction of conditioned fear responses. ${ }^{3}$ This may cause intense anxiety, as well as problems with the normal age-related development of behavioral and emotional regulation (including the inhibition of impulsive behaviors). ${ }^{3}$

\section{- Superior temporal gyrus and social intelligence}

The neural basis of our abilities to understand the feelings of other people is through the amygdala and its projections to the superior temporal gyrus, thalamus, and to the prefrontal cortex. ${ }^{3}$ This is called the theory of mind or social intelligence. ${ }^{3}$ Maltreated children with PTSD were found to have more superior temporal gyrus gray matter. ${ }^{3}$ This may be the result of a developmental deficit in the age-appropriate pruning of the superior temporal gyrus ${ }^{3}$, or it may be the basis of the increased social intelligence found in abused children. ${ }^{22}$

\section{- Cerebellar vermis}

The cerebellum comprises only 10-20\% of brain volume, yet contains over $50 \%$ of the neurons. ${ }^{17}$ Of all brain regions, the cerebellum shows the greatest postnatal growth. ${ }^{17}$ The cerebellum possesses the highest density of glucocorticoid receptors, even exceeding that of the hippocampus, and appears to be important in mediating the response to stress. ${ }^{17}$

The vermis of the cerebellum receives input from the vestibular nuclei and is important for multisensory integration and the control of epilepsy and limbic activation. ${ }^{17}$

Proprioceptive and vestibular stimulation is essential for the normal development of the vermis. ${ }^{17}$ The cerebellar vermis strongly influences the locus coeruleus, ventral tegmental area and substantia nigra, and thus the primary norepinephrine and dopamine projections. ${ }^{17}$ In primates, the adverse effect of rearing without maternal contact was 
significantly attenuated by vestibular stimulation from a rocking wire monkey surrogate. ${ }^{17}$ Abnormalities in the cerebellar vermis may contribute to cognitive, linguistic, social and emotional disturbances and are implicated in a variety of psychiatric disorders, including autism, schizophrenia, ADHD, bipolar and unipolar depression. ${ }^{17}$ Insufficient rocking and stroking by the primary caregiver may thus lead to underdevelopment of the cerebellar vermis and predispose to psychopathology. ${ }^{17}$

Having described the neuroendocrinological consequences of childhood maltreatment, we turn to the debate on whether these changes are pathological or adaptational.

\section{Pathology or adaptation?}

Initially, the neuroendocinological results of child maltreatment were thought to reflect pathology. Subsequently researchers started to consider whether the symptoms of psychopathology could reflect 'normal' reactions to abnormal events. ${ }^{21}$ Recently Teicher et al. proposed an alternative evolutionary hypothesis: maybe early stress triggers the brain to develop along an alternative pathway in order to "survive and reproduce in a malevolent stress-filled world". ${ }^{17}$ The ability to mount an intense fight-or-flight response and react aggressively on the slightest provocation may be adaptive in the short-term in a hostile world. ${ }^{17}$ However, these changes are not favorable on the longterm and may predispose to the risk of developing serious medical and psychiatric disorders. ${ }^{17}$

\section{Conclusion}

In theory, child maltreatment is preventable. It would be more efficient to avert damage of the developing brain rather than to try to correct it later. However, in reality this is unfortunately often not feasible. Removing children from homes in which they are maltreated may establish continuing patterns of abuse and neglect as social services are not always able to meet the demands of children in care. It is important to note that antisocial behavior in adults is linked to both past and future childhood maltreatment and violence. ${ }^{14}$

Fortunately, children's brains remain plastic and capable of growth and development. ${ }^{12}$ The ability to exercise control over their actions, and self-organization should be encouraged in those children who are reached by social services. ${ }^{1,3}$ Also, adult survivors of childhood abuse may learn strategies for selfregulation of disturbed emotional and physiological systems, e.g. dialectical behavior therapy or mentalising treatment. ${ }^{23,24}$ The consequences, and outcomes, of childhood maltreatment are influenced by a complex interaction of environmental and individual factors. It would be prudent for such knowledge to be translated into meaningful and adequately resourced policies and legislation that facilitates appropriate interventions at both a societal and individual level.

\section{References}

1. Glaser D. Child abuse and neglect and the brain--a review. J Child Psychol Psychiatry. 2000 Jan;41(1):97-116.

2. Flinn MV. Evolution and ontogeny of stress response to social challenges in the human child. Developmental Review. Vol 26(2) Jun 2006, 138-174.

3. De Bellis MD. The psychobiology of neglect. Child Maltreatment 2005;10(2):150-172.
4. Charmandari E, Kino T, Souvatzoglou E, Chrousos GP. Pediatric stress: hormonal mediators and human development. Horm Res. 2003;59(4):161-79.

5. Susman EJ. Psychobiology of persistent antisocial behavior: Stress, early vulnerabilities and the attenuation hypothesis. Neurosci Biobehav Rev 2006:30(3):276-389

6. Sapolsky RM, Krey LC, McEwen BS. The neuroendocrinology of stress and aging: The glucocorticoid cascade hypothesis. Endocr Rev 1986;7:284-301.

7. Sirianni JP. Psychological Stress and Language Processing in SchoolAged Children. Journal of Speech Language Pathology and Audiology. Vol 28(3) Fall 2004, 112-121

8. Huizink AC, Robles de Medina PG, Mulder EJH, Visser GHA, Buitelaar JK Stress during pregnancy is associated with developmental outcome in infancy. J Child Psychol Psych 2003;44(6):810-818.

9. King $S$, Laplante DP. The effects of prenatal maternal stress on children's cognitive development: Project Ice Storm. Int J Biol Stress 2005;8(1):3545.

10. Jarvis KL, Gordon EE, Novaco RW. Psychological Distress of Children and Mothers in Domestic Violence Emergency Shelters. Journal of Family Violence. Vol 20(6) Dec 2005, 389-402.

11. Blair C. How similar are fluid cognition and general intelligence? A developmental neuroscience perspective on fluid cognition as an aspect of human cognitive ability. Behav Brain Sci 2006:29(2):109-160.

12. Noble KG, Tottenham N, Casey BJ. Neuroscience perspectives on disparities in school readiness and cognitive achievement. Future Child. 2005 Spring; 15(1):71-89.

13. Asbury K, Wachs TD, Plomin R. Environmental moderators of genetic influence on verbal and nonverbal abilities in early childhood. Intelligence 2005,33(6):643-661.

14. Heide KM, Solomon EP. Biology, childhood trauma, and murder: Rethinking justice. Int J Law Psych 2006:29(3);220-233.

15. Tsuang M. Schizophrenia: genes and environment. Biol Psychiatry 2000;47(3):210-220

16. Kaplow JB, Widom CS. Age of onset of child maltreatment predicts long-term mental health outcomes. J Abnorm Psychol 2007; 1 16(1):176187

17. Teicher MH, Andersen SL, Polcari A, Anderson CM, Navalta CP, Kim DM The neurobiological consequences of early stress and childhood maltreatment. Neurosci Biobehav Rev 2003;27(1-2):33-44.

18. Bremner JD, Vythilingam M, Vermetten E, Adil J, Khan S, Nazeer A, Afzal $N$. Cortisol response to a cognitive stress challenge in posttraumatic stress disorder (PTSD) related to childhood abuse. Psychoneuroendocrinology. 2003 Aug;28(6):733-50.

19. Kajantie E, Phillips DI. The effects of sex and hormonal status on the physiological response to acute psychosocial stress. Psychoneuroendocrinology. 2006 Feb;31 (2):151-78.

20. Navalta CP, Polcari A, Webster DM, Boghossian A, Teicher MH. Effects of childhood sexual abuse on neuropsychological and cognitive function in college women. J Neuropsych Clin Neurosci 2006;18(1):45-53.

21. Caffo E, Forresi B, Lievers LS. Impact, psychological sequelae and management of trauma affecting children and adolescents. Current Opinion in Psychiatry. Vol 18(4) Jul 2005, 422-42.

22. Naude H, du Preez CS, Pretorius E. The impact of child abuse as traumatic environmental stressor on the plasticity of intelligence. CARSA 2003;4(2):10-26.

23. Linehan MM, Armstrong HE, Suarez A, Allmon D, Heard HL. Cognitivebehavioral treatment of chronically parasuicidal borderline patients. Arch Gen Psychiatry 1991;48(12):1060-1064.

24. Bateman AW, Fonagy P. Effectiveness of psychotherapeutic treatment of personality disorder. Br J Psychiatry 2000;1 177:138-43. 